\title{
Counterpropagation fuzzy-neural network for city flood control system
}

\author{
Fi-John Chang ${ }^{a, *}$, Kai-Yao Chang ${ }^{a}$, Li-Chiu Chang ${ }^{b}$
}

\author{
a Department of Bioenvironmental Systems Engineering, National Taiwan University, No. 1, Sec. 4. Roosevelt Road, Taipei \\ 106, Taiwan, ROC \\ ${ }^{\mathrm{b}}$ Department of Water Resources and Environmental Engineering, Tamkang University, Taiwan, ROC
}

Received 28 September 2007; received in revised form 7 May 2008; accepted 10 May 2008

KEYWORDS
Fuzzy-neural network;
Rule-base control;
Artificial intelligence;
Flood;
Pumping station
operation

\section{Introduction}

Due to urbanization and climate change, existing drainage systems are facing a great challenge. It is imperative to construct an effective and efficient flood control system and

\footnotetext{
${ }^{*}$ Corresponding author. Tel.: +886 2 23639461; fax: +886 2 23635854.

E-mail address: changfj@ntu.edu.tw (F.-J. Chang).
}

\begin{abstract}
Summary The counterpropagation fuzzy-neural network (CFNN) can effectively solve highly non-linear control problems and robustly tune the complicated conversion of human intelligence to logical operating system. We propose the CFNN for extracting flood control knowledge in the form of fuzzy if-then rules to simulate a human-like operating strategy in a city flood control system through storm events. The Yu-Cheng pumping station, Taipei City, is used as a case study, where storm and operating records are used to train and verify the model's performance. Historical records contain information of rainfall amounts, inner water levels, and pump and gate operating records in torrential rain events. Input information can be classified according to its similarity and mapped into the hidden layer to form precedent if-then rules, while the output layer gradually adjusts the linked weights to obtain the optimal operating result. A model with increasing historical data can automatically increase rules and thus enhance its predicting ability. The results indicate the network has a simple basic structure with efficient learning ability to construct a human-like operating strategy and has the potential ability to automatically operating the flood control system.
\end{abstract}

(C) 2008 Elsevier B.V. All rights reserved. drainage mechanism. Taiwan is located in the north Pacific Ocean subtropical jet stream monsoon district. Taipei City, the capital of Taiwan, is located in the Taipei Basin in northern Taiwan and is surrounded by the Danshui River, with a narrow estuary that makes it difficult for water in the city to be effectively discharged. A high intensity rainfall during a storm or typhoon could easily cause flooding. The Nari typhoon struck North Taiwan with stunning rainfall on September 17th 2001, where the accumulated precipitation in 
Taipei City plain area was 500 mm/day and in mountain area was over $1000 \mathrm{~mm}$; the highest precipitation reached $148.5 \mathrm{~mm} / \mathrm{h}$. It caused 27 deaths, with 3 missing, and 16 injured. Taipei mass rapid transportation (MRT) system was flooded (all underground lines were just like sewers, including 16 stations, the heavy-capacity system operation control center, and the administration building), 4151 building basements were inundated, the city functions were severely damaged, and the economic effects were countless.

In these circumstances, the flood control strategy for Taipei City was to build an embankment along the river side to prevent a two-hundred year river flood from entering the city and to set up pumping stations at the main draining lines to pump out the inner city water. Undoubtedly, the pumping stations play an important role in the Taipei flood controlling system. A pumping station that fails to perform its designed draining function can cause flood disasters and result in resentment from the residents and their loss of property. Related operating guidelines were built a long time ago, where the hydrological data were lacking and with high uncertainty. The pumping stations have been operated under actual circumstances and require experienced operators to adjust the switching (turning on and off) of the pump machines according to the actual precipitation and the changes of fore bay levels. To overcome the disadvantages of traditional operating guidelines, which are difficult to follow due to ambiguous forms and the need for skilful operators, we propose a rule-based fuzzy-neural network to effectively operate the pumping sets.

The last decade has witnessed growing interest in the use of fuzzy logic and neural networks in the field of hydrological modeling (Chang et al., 2001; Xiong et al., 2001; Lobbrecht and Solomatine, 2002; Bhattacharya et al., 2003; Jingyi and Hall, 2004; Nayak et al., 2004; Lobbrecht et al, 2005; Chang and Chang, 2006; Lohani et al., 2006; Tutmez et al., 2006; Bae et al., 2007; Chaves and Kojiri, 2007; Hsu and Wei, 2007). The neural networks and fuzzy systems are either used as competing alternatives to the traditional hydrological models or work in synergy with the traditional models to produce better modeling solutions. Based on the realization that a fuzzy logic is a nonlinear mapping from an input space to an output space and a neural network can be adapted to data, a fusion of fuzzy logic and neural network model could solve the highly non-linear control problem robustly and effectively (Chang and Chang, 2001). To bridge the gap between academic research and actual operation, we propose an artificial intelligent technology that fuses the ANN and fuzzy logic features for extracting the flood control knowledge for the Taipei city flood control system. The major advantage of this fuzzy-neural network is that it can tune the complicated conversion of human intelligence to a fuzzy logical operating system. The network is constructed by adopting the historical hydrological data of storm events, and the historical operating records of the gravity gates and pump sets, with the judging method of human beings. The detailed methodology and the results are provided in the following sections.

\section{Theoretical background}

Artificial Intelligence is a state-of-the-art technology that resembles the human thinking process in decision making and strategy learning, and it has been well recognized for its outstanding ability in controlling complex systems, such as reservoir operating systems (Chang et al., 2005; Chaves and Chang, 2008). The ANN and fuzzy logic are two effective tools in developing an intelligent control engineering model. Fuzzy logic is controlled by a human operator to deal with reasoning that is approximate rather than precisely deduced from classical predicate logic. In the absence of a system mathematical model, fuzzy logic is analogous to human behaviour to establish the relationships between the inputs and the outputs, based on approximate reasoning bound by a minimum set of rules. An artificial neural network (ANN) is an interconnected group of neurons that changes its structure and connected weights based on external or internal information that flows through the network. ANN can be used to model complex relationships between inputs and outputs. Neural networks are trainable systems that can "learn" to solve complex problems from a set of exemplars and generalize the "acquired knowledge" to solve unforeseen problems as in environmental prediction. Today ANNs are being applied to an increasing number of real-world problems of considerable complexity.

This study is based on a rule base, fuzzy control, and counterpropagation neural network to establish the proper relationship between hydrological variations and pump station operation. Fuzzy-Neural Network, which was first introduced by Nie and Linkens (1994), Nie and Linkens (1995), consists of two parts, where the first part is clustering the input data and the second part is a look-up table for mapping input and output patterns. The architecture of the counterpropagation fuzzy-neural network (CFNN) consists of three layers: input, hidden (Kohonen), and output (Grossberg) layers (Fig. 1). The input layer with $n$ nodes stores the input values, the Kohonen layer with $p$ nodes clusters the input values with a similarity measurement, and the Grossberg layer with $m$ nodes calculates the outputs by summing the weighted Kohonen layer outputs.

The CFNN has a hybrid learning scheme, unsupervised and supervised, to learn human beings' experiences and operating rules. Unsupervised learning is used during the first stage for clustering the input vectors to separate distinct sets according to the similarity of nodes and formed precedent if-then rules. During the second stage of learning, the weight vectors between the Kohonen and Grossberg layers are adjusted by supervised learning to reduce the errors. A model with increasing historical data can automatically increase rules and thus enhance its predicting ability. The CFNN has efficient clustering effects from human-like ability of extracting rules, and good simulating results from non-linear functions. It is a good pattern recognition engine and robust classifier, with the ability to generalize in making decisions about imprecise input data. Details of the CFNN procedure can be found in Chang et al. (2001) and are summarized as follows.

\section{Network learning algorithms}

A network input vector $X=\left(x_{1}, \ldots, x_{i}, \ldots, x_{n}\right)$ would be transformed to an output vector $Y=\left(y_{1}, \ldots, y_{m}\right)$ through a logical judgment, which is called a set of rule. The connection of the input layer to the nodes in the Kohonen layer is $w$, which is an 'IF' statement of rule-base control, and the 


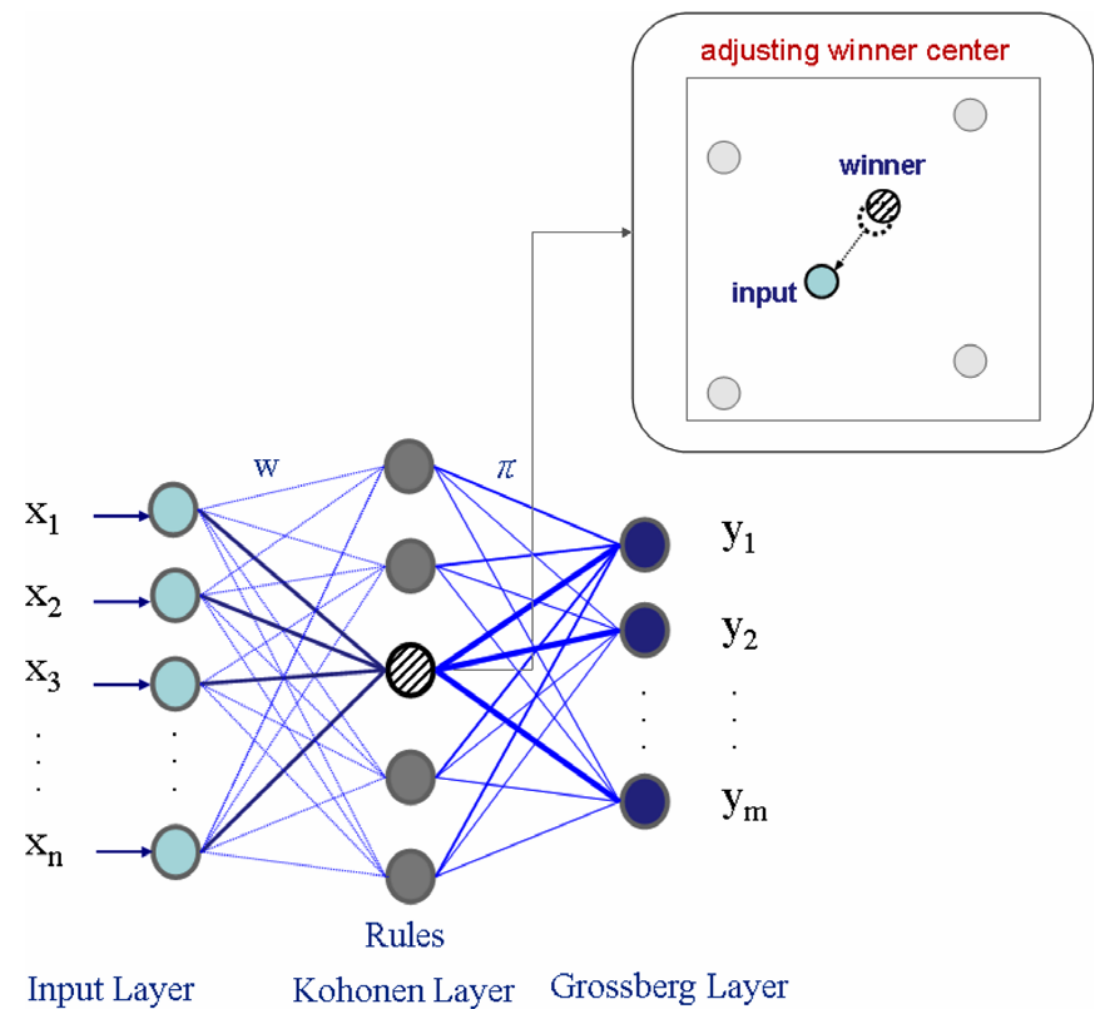

Figure 1 Representation of a counterpropagation fuzzy-neural network.

next connection to the nodes in the Grossberg layer is $\pi$, which is the 'THEN' statement of rule-base control. Thus, the two-step connections could be viewed as a whole 'IF $X$ is $w$, THEN $Y$ is $\pi^{\prime}$ statement. The weights in the Kohonen and Grossberg layers are trained independently and shown as follows.

\section{Kohonen layer}

In the Kohonen layer, every node represents a rule unit. The number of nodes in this layer is the same as the number of rules. We have to decide the number of rules and the center of each rule unit $w_{j}$. First, the learning arithmetic computes the distance between the input $X(t)$ with the center $w_{j}$ of the existing $N$ nodes and decides the winning node (the shortest distance). Then, the weights of winner node will be adjusted in the following condition.

If the closest distance between $w_{j}$ and input $X(t)$ is smaller than $\Delta$ ( $\Delta$ is the interval in a triangular function), then the $w_{j}$ is updated by the following equation:

$w_{j}^{\text {new }}=w_{j}^{\text {old }}+\alpha\left[X(t)-w_{j}^{\text {old }}\right]$

where $X(t)$ is the $t$ th input vector, and $\alpha$ is the learning rate, which is between 0 and 1 .

If the distance between $w_{j}$ and $X(t)$ is larger than $\Delta$, a new rule $w_{j}^{\text {new }}=X(t)$ will be created, and the existing $N$ rules will become $(N+1)$ rules (Kohonen, 1998). In the arithmetic, when the $\Delta$ value decreases, the number of units in the rule base and the accuracy (especially in train set) will increase and the complexity of the network will also increase. The decision has to be made between accuracy and model complexity. Generally, $\Delta$ is selected by trialand-error method.

\section{Grossberg layer}

The weights $(\pi)$ in this layer is updated according to the Grossberg learning rule, as follows:

$\pi_{j}^{\text {new }}=\pi_{j}^{\text {old }}+\beta\left[Y(t)-\pi_{j}^{\text {old }}\right]$

where $Y(t)$ is the output vector, and $\beta$ is the learning rate, which is between 0 and 1 . Only the weights connecting the winning neuron in the Kohonen layer will be updated.

\section{Network predicting}

The network-predicting algorithm consists of two steps, pattern matching and weighted average determining, and is coupled with the concept of fuzzy control (Nie, 1997). Pattern matching takes the Gaussian function as membership function $\left(s_{j} \in[0,1]\right)$ for calculating the similarity of the input value and the $j$ th rule unit. If the central vector distance between input and the $j$ th rule is larger than $\Delta$, then $s_{j}=0$, which means the influence weight of the $j$ th rule to the input is 0 .

The weighted average is used to calculate the fuzzy output $\hat{Y}(t)$ as follows.

$Y \wedge(t)=\frac{\sum_{j=1}^{N} s_{j} \pi_{j}}{\sum_{j=1}^{N} s_{j}}$

where $\hat{Y}(t)$ denotes the fuzzy output of $X(t), \pi_{j}$ is the output weight. 
After the network is trained, all of the weights are kept fixed. Only input data is needed for the model to operate for predicting.

In practice, the input $X(t)$ might not be clustered into any rule because the distances of each rule's center are all larger than the interval $\Delta$. The reason could be a lack of historical data for training network, or the value of $\Delta$ is too small, which causes the data not to be grouped properly and effectively. One solution is trying to find a suitable $\Delta$ that makes the model have proper clustering abilities. The other way is to enlarge the existing width of $\Delta$ by a geometric ratio that increases the opportunity of clustering the rule units (Chang et al., 2001). In this study, the latter solution is implemented into the proposed CFNN for modelling the pumping station operation.

\section{Case study}

The city of Taipei and its suburbs, bounded by mountains, was the site of a great lake in prehistoric times; it slowly developed its present features as alluvial deposits filled the lake. Topographically, the Danshui River and its tributaries surround the Taipei basin and its outlet, the Guandu pass, near estuary is narrow, so that running water in the city cannot be discharged by gravity when the river level reaches a certain height as storms strike Taipei in a monsoon. The flood control strategy has built an embankment along the river side and set pumping stations at main drain lines to discharge the rainfall in the city area.

\section{Yu-Cheng pumping station}

The Yu-Cheng pumping station is located in the east of Taipei City. The total measure of the watershed is 1644.93 ha. Fig. 2 shows its geographic location and Fig. 3 shows its watershed. The station was completed in 1987 to pump inner water to the Keelung River. It contains seven massive pumps whose pumping capacity is $26.3 \mathrm{~m}^{3} / \mathrm{s}$ each and that makes the total capacity to $184.1 \mathrm{~m}^{3} / \mathrm{s}$. In the 1980 s the station was said to be the most advanced and largest in Asia. Its facilities are shown in
Fig. 4. The start-out level is EL. $2.4 \mathrm{~m}$. Design outer-level is the 10 -year flood level, EL. 7.62 m, of Keelung River. The current capacity can only drain away the rainfall runoff of a rainfall intensity of $42.5 \mathrm{~mm} / \mathrm{h}$. According to the watershed of main drainage lines, the system of the Yu-Cheng pumping station could be roughly divided as Songlong Road line system, Zhongpo North Road line system and Nangang Road line system, 3 sub-drainage systems. There is a level monitoring booth, Yu-Cheng 10 (YC10) in the watershed of Yu-Cheng pumping station.

The gravity gates of Yu-Cheng pumping station usually remain open to drain out the inner water to the Keelung River. When the level of the river tends to rise up to the warning level of the fore bay of the pumping station, the pumps turn and warm up. The pumps start pumping as soon as the water level is up to the start-out level and the gravity gates are closed at the same time. The 7 pump sets are operated independently and sequentially (see Fig. 5).

\section{Pumping station operation}

\section{Operation target}

Mechanical power is used to drain away the inner water as the outer level is higher than the inner level and the running water cannot be discharged by gravity. The pumped water will lower the level of the drainage system before the flood peak to increase the storage capacity of the system and to speed up the discharging for decreasing the flooding probability.

\section{Current operation mechanisms (Taipei City Government, 1989, 1998; Pumping Station Operation Manual et al., 1984)}

There are several standards for guiding the pumps to turn on or off. The minimum water level is the lowest water level for pumps functioning; the pumps may be damaged if the water is lower than this level. The pumps are warmed up and stand-by as the water level reaches the warning water level. The start-out water level is the pumps starting to function at this water level. Generally, the pumps start sequentially, which means when a running pump cannot

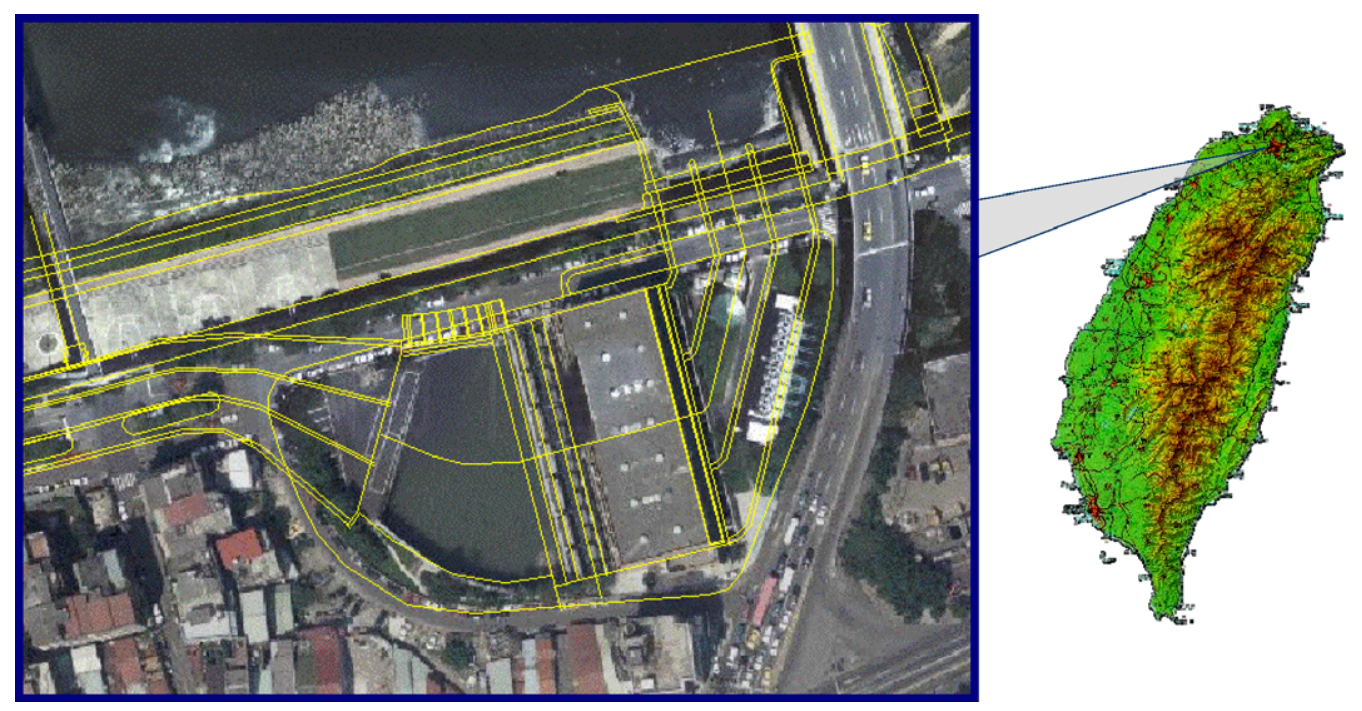

Figure 2 Location of Yu-Cheng pumping station, Taipei, Taiwan. 


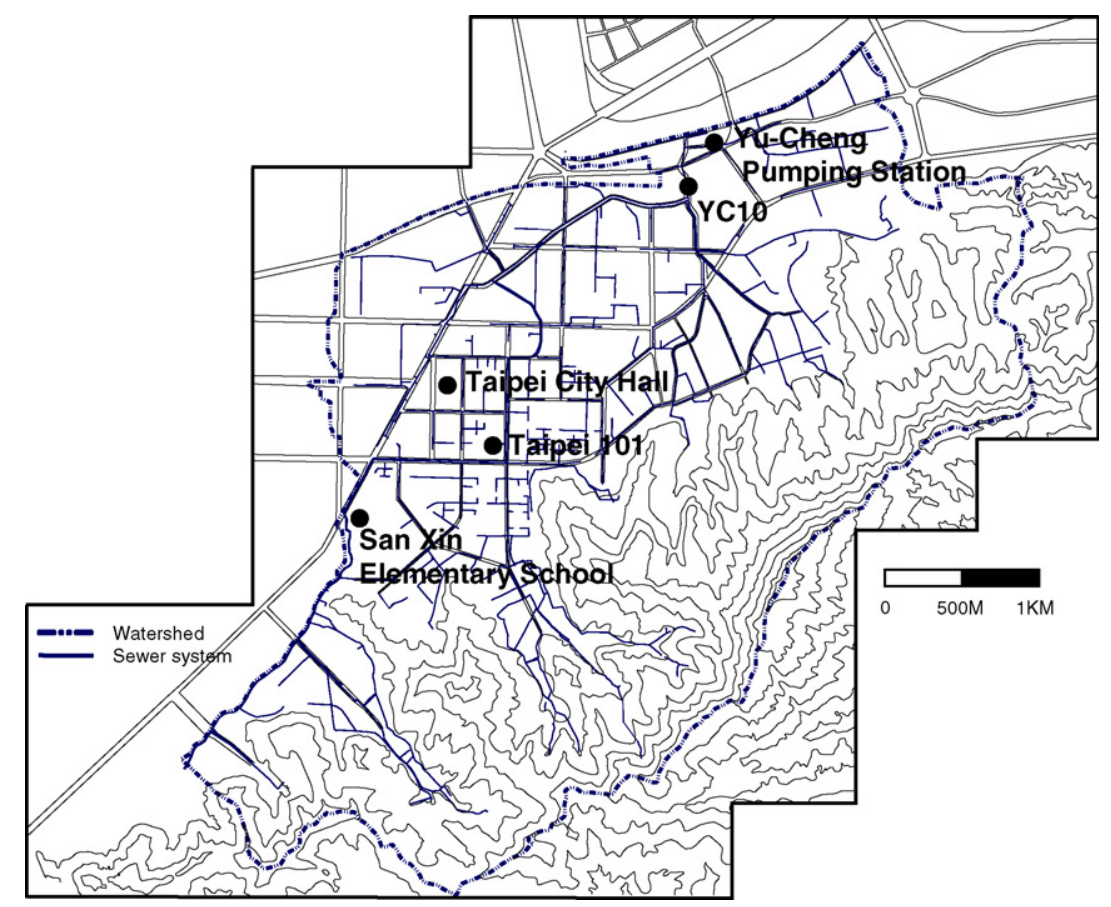

Figure 3 Watershed of the Yu-Cheng pumping station.

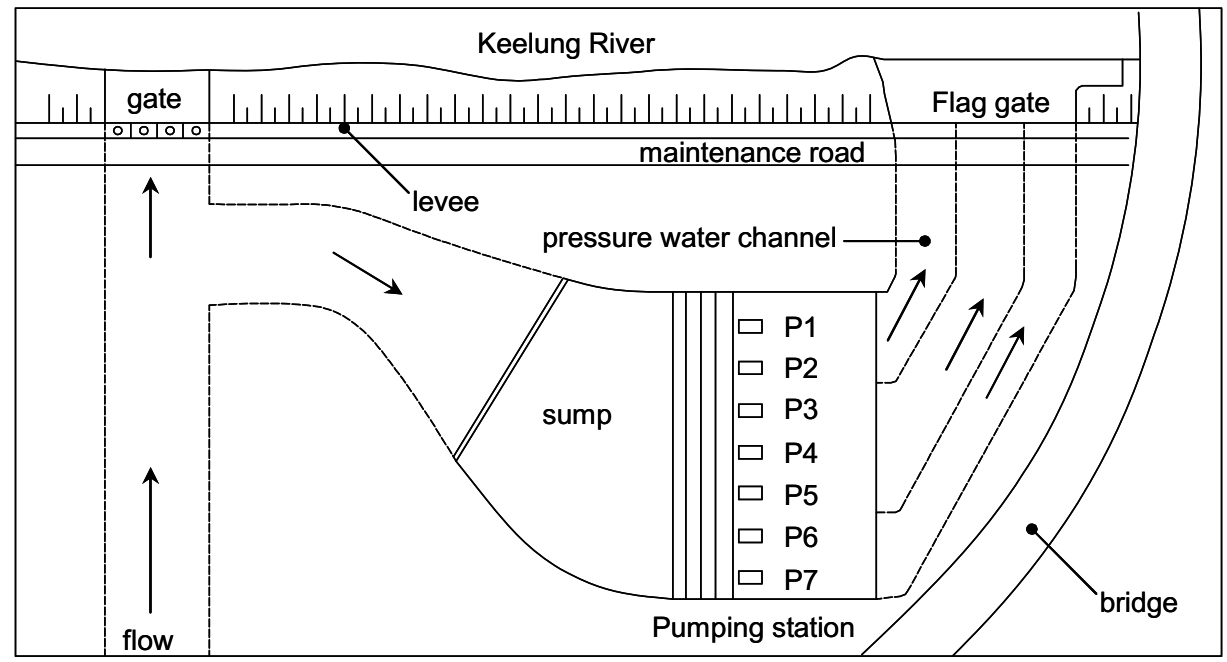

Figure 4 Diagram of the Yu-Cheng pumping station.

control the water level under the start-out level, another pump will start working. On the contrary, the pumps are turned off, respectively, as the water level is falling. The maximum water level is the maximum defensible water level in the pumping station.

As the river level rises to the bottom of the gravity gates, the gates are closed immediately to prevent the flow in. Since the gravity gates are closed, runoff in the city cannot be discharged into the river, causing the level of fore bay to rise. If the level were to reach the warning level, the pump sets would be warming up and would start to pump water when the level reached the start-out level. The gravity gates should remain closed while the pump sets keep draining water. When the river level is lower than the fore bay level, the gravity gates are opened immediately to speed up the drainage. In addition, if the level of fore bay is still higher than the start-out level, the pump sets keep running.

\section{Pumping operation factors analyses}

The operating decision mainly depends on the variation of fore bay level caused by rainfall runoff. Their relationship, however, is very complex. Therefore, the rainfall situation, the variation of fore bay level, the rainfall in the future, and fore bay level prediction are all important factors that influence the decision. As mentioned above, the operation of gravity gates is considerably related to the operation of pump sets, the changes of inner level, and the comparison of inner and outer levels. 


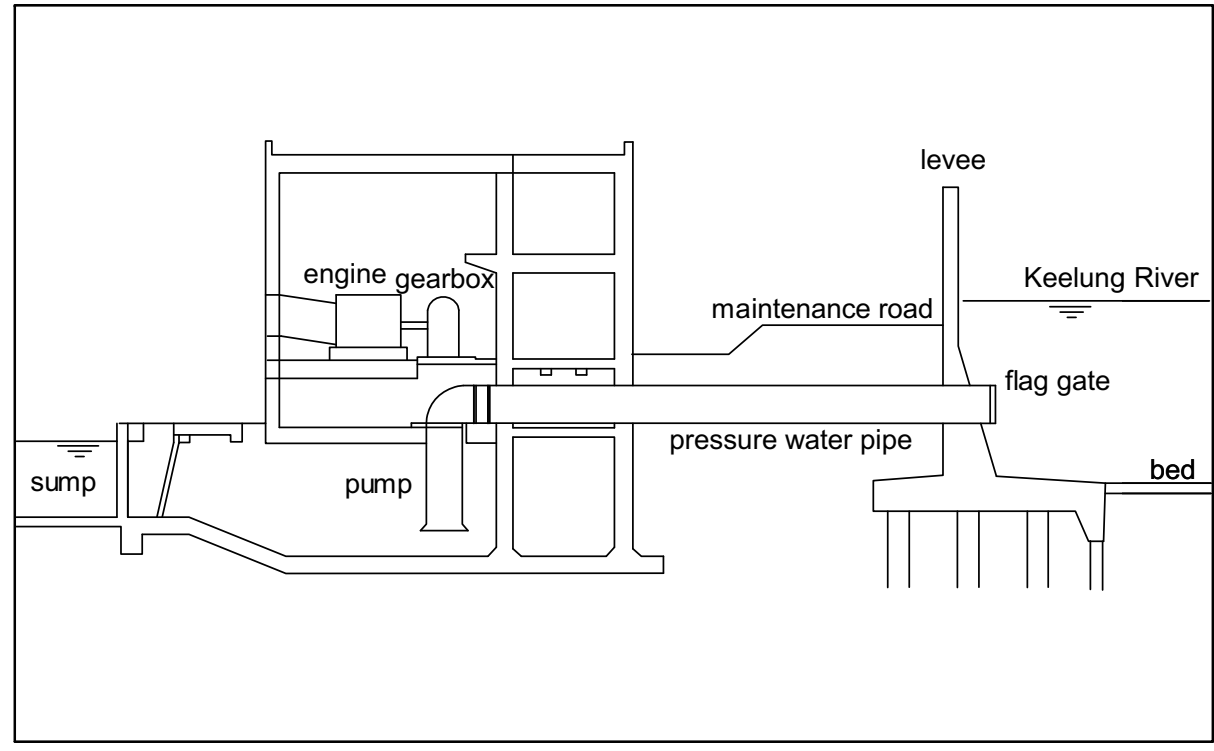

Figure 5 Section of pumping station facilities.

To sum up, the purpose of the pumping station operation is to efficiently lower the fore bay level, keep it under the maximum tolerable level to avoid flooding but not under its minimum tolerable limits to avoid damaging to pump sets. And at the same time, the pump sets should not be turned on and off too frequently during the period of operation to keep the pumps from being damaged.

\section{Network model and parameter settings}

To build up an intelligent control system, one first needs to acquire knowledge of the system. The knowledge could be rules or guidelines, which explicitly show the way that a system responds to an input. Unfortunately, in this case no strict rules exist to guide the way. One alternative way is to collect the historical operating records (i.e., the inputoutput patterns), and then try to analyze the input-output relation. If a solid relation can be found, it can be used for future operation when we face a similar input situation. The proposed intelligent flood control system learns from the decisions made by the experienced operators, which are considered to be the optimal decisions in this study.

We collected the precipitation, fore bay level, opening and closing of the gravity gates, and historical records of pump set operation from seven storm events. The precipitation variation was treated by accumulated precipitation of different duration (such as 5-min accumulated precipitation, 10-min accumulated precipitation). The fore bay level variation was treated by the lifts of different duration (the difference between current and $5 \mathrm{~min}$ before). Since it

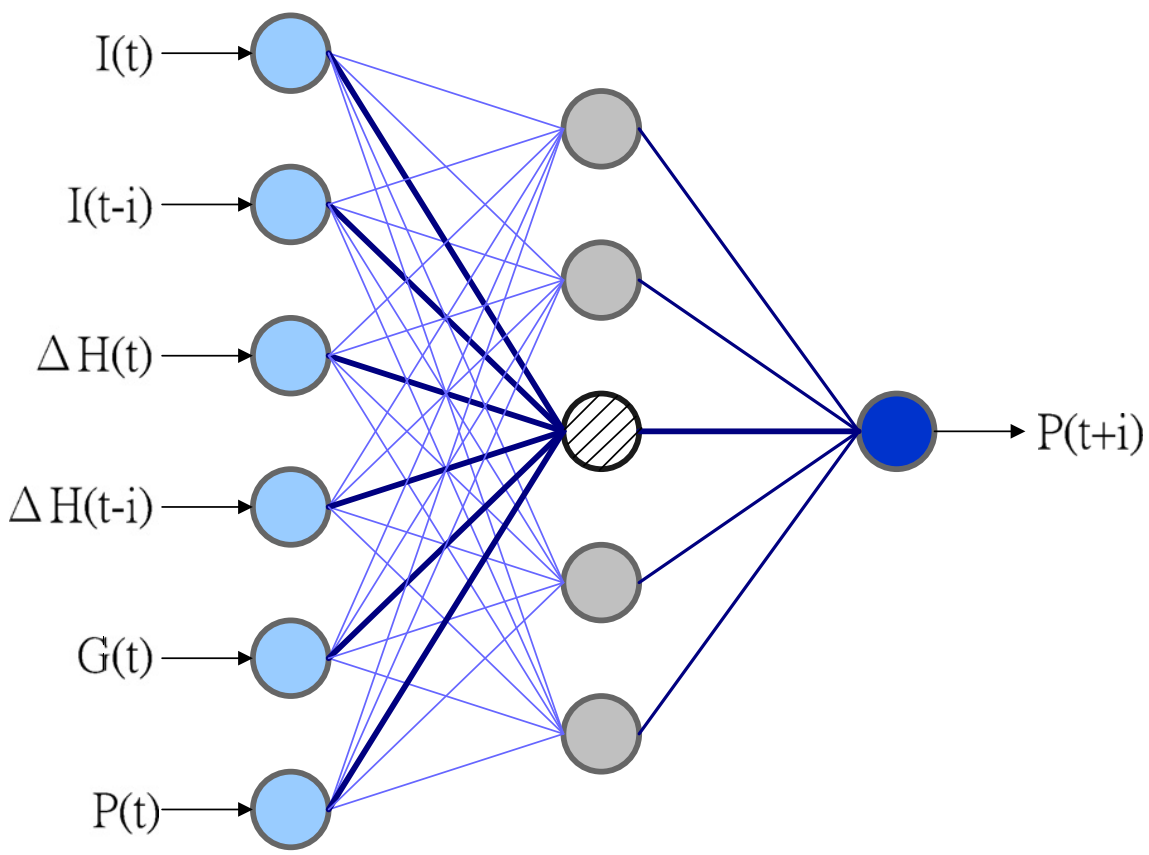

Figure 6 Schematic diagram of CFNN for pumping station operation. 
Table 1 Chosen data of typhoons and floods events in Yu-Cheng pumping station

\begin{tabular}{|c|c|c|c|c|c|c|c|c|}
\hline \multirow[t]{2}{*}{ Stage } & \multirow[t]{2}{*}{ Events } & \multirow[t]{2}{*}{ Starting time } & \multirow[t]{2}{*}{ Ending time } & \multirow[t]{2}{*}{$\begin{array}{l}\text { Duration } \\
\text { (min) }\end{array}$} & \multirow{2}{*}{$\begin{array}{l}\text { Accumulation } \\
\text { of precipitation } \\
(\mathrm{mm})\end{array}$} & \multicolumn{3}{|c|}{$\begin{array}{l}\text { Maximus precipitation } \\
(\mathrm{mm})\end{array}$} \\
\hline & & & & & & $5 \mathrm{~min}$ & $10 \min$ & $1 \mathrm{~h}$ \\
\hline \multirow[t]{5}{*}{ Training } & Storm Event & $2002 / 12 / 2102: 34$ & $2002 / 12 / 21$ 10:05 & 451 & 37.0 & 6.5 & 13.0 & 35.0 \\
\hline & Storm Event & $2002 / 12 / 2517: 59$ & $2002 / 12 / 25$ 20:41 & 162 & 20.0 & 1.5 & 2.0 & 7.0 \\
\hline & Storm Event & $2002 / 12 / 2605: 27$ & $2002 / 12 / 2611: 16$ & 349 & 45.0 & 8.5 & 13.5 & 37.0 \\
\hline & Haima Typhoon & $2004 / 9 / 10$ 16:39 & $2004 / 9 / 11$ 13:51 & 1282 & 445.5 & 11.0 & 18.0 & 74.0 \\
\hline & Storm Event & $2006 / 9 / 1023: 54$ & $2006 / 9 / 1121: 55$ & 1321 & 58.0 & 2.5 & 5.0 & 15.5 \\
\hline Validation & Storm Event & $2007 / 6 / 914: 00$ & $2007 / 6 / 9$ 17:35 & 515 & 24.0 & 3.0 & 4.0 & 8.5 \\
\hline Testing & Haitang Typhoon & $2005 / 7 / 17$ 23:21 & $2005 / 7 / 18$ 18:49 & 1168 & 170.0 & 5.5 & 6.5 & 32.5 \\
\hline
\end{tabular}

would cause damage to the pump sets if the machines were turned on and off too frequently, the maximum number of such operations is normally set as 12 times in an hour. The precipitation and water level differences were normalized into the interval between 0 and 1 before they were put in the network.

The input layer consists of the accumulated precipitation of current and previous time, $I(t)$ and $I(t-i)$; the differences of fore bay level at two time, $\Delta H(t)$ and $\Delta H(t-i)$; the number of closing gates at current time, $G(t)$; and the number of active pumps at current time, $P(t)$. And the output layer is the number of active pumps at next $5 \mathrm{~min}, P(t+i)$ in this case $i=5$. Those inputs and output are shown as Fig. 6 . The precipitation caused by a typhoon or storms during the wet season can be huge (e.g., $100 \mathrm{~mm} / \mathrm{h}$ is not unusual) while the capacity of the fore bay is relatively limited; consequently, the fore bay water level would change rapidly. The model can be running every minute to predict the number of running pumps in the next 5-min for the operating center as the latest information, so the possible variations can be estimated ahead of time and the operators will have more time to make a suitable decision.

To construct a suitable neural network, the data sets are divided into three independent sub-sets, namely training, validation, and testing subsets. The training sets are used to adjust the connected weights of the constructed networks, the validation sets are implemented to select a suitable network from the above-trained networks, and the reliability of the selected network is then tested by testing sets. In this study, we arbitrarily divided event datasets into training, validation and testing subsets. The data adopted for training are the data of 5 storm events (total 3565 data sets), and thus the rule base is built. Then the data of one event in 2007 (515 data) are used for validation. After that, the data of Haitang Typhoon (1168 data) are used for testing. Table 1 shows the chosen data for modelling the networks.

There are two learning rates ( $\alpha$ and $\beta$ ) and one matching parameter $(\Delta)$, which need be predetermined before the network is trained. The mean average error (MAE) is taken as the criterion to judge the error between the modelling predicting values and the actual operating values. A smaller MAE represents higher accuracy. The formulations of MAE are shown as below:

MAE $=\frac{\sum_{t=1}^{N}\left|P_{\text {est }}(t)-P_{\text {obs }}(t)\right|}{N}$ where $N$ is the number of times, and $P_{\text {est }}$ and $P_{\text {obs }}$ are the number of active pumps by model estimation and observed (human operated) record.

\section{Observed rain, water level and the pumping station operation information}

The real-time water level monitoring of the sewerage systems, which is very helpful for flood alarming systems, has been operated by the hydraulic engineering office (HEO) of Taipei City Government in last few years. The data used in this study were built since 2002 near the fore bay of YuCheng pumping station. The seven recorded typhoons and/ or storm events are shown in Table 1. The precipitation data were obtained from the San Xin Elementary School station, which is located in the watershed of Yu-Cheng Pumping Station (shown in Fig. 3) also operated by the HEO.

\section{Results and analyses}

The initial settings for training the model are $\alpha=0.5$ and $\beta=0.5$, the limit of fault tolerance is 0.0001 . The initial va-

Table 2 Summary of the performance of the different $\Delta$ at different stages

\begin{tabular}{lrlll}
\hline$\Delta$ & Rules & MAE & & \\
\cline { 3 - 5 } & & Training & Validation & Testing \\
\hline 0.04 & 465 & 0.151 & 0.404 & 0.433 \\
0.06 & 229 & 0.189 & 0.548 & 0.351 \\
0.08 & 158 & 0.252 & 0.552 & 0.434 \\
0.1 & 107 & 0.289 & 0.452 & 0.384 \\
0.12 & 84 & 0.254 & 0.206 & 0.081 \\
0.14 & 66 & 0.288 & 0.194 & 0.104 \\
0.16 & 49 & 0.458 & 0.368 & 0.312 \\
0.18 & 41 & 0.591 & 0.450 & 0.294 \\
0.2 & 35 & 0.217 & 0.122 & 0.049 \\
0.22 & 32 & 0.355 & 0.142 & 0.223 \\
0.24 & 21 & 0.395 & 0.152 & 0.082 \\
0.26 & 21 & 0.255 & 0.138 & 0.147 \\
0.28 & 15 & 0.393 & 0.136 & 0.050 \\
0.3 & 13 & 0.420 & 0.142 & 0.095 \\
0.4 & 6 & 0.757 & 0.792 & 0.409 \\
0.5 & 4 & 1.102 & 0.804 & 0.893 \\
\hline
\end{tabular}


lue of $\Delta$ is set as 0.04 and is increased 0.02 in each constructed network until $\Delta$ reaches 0.5 . Consequently, 24 networks were trained and evaluated. The influence of the $\Delta$ value in the training phase of network constructing and in the validation and test phases of network performance is shown in Table 2 and Fig. 7. As expected, we found that in training stage a smaller $\Delta$ will provide smaller predicting error, while in validation and testing stages, after a certain value of the $\Delta$, the predicting results cannot be improved further and the accuracy is decreased instead. For instance, when $\Delta$ is 0.04 , there are 465 rules units and the value of
MSE in training stage is quite ideal. When $\Delta$ is 0.5 , only 4 rules units are built; however, larger error was obtained. It is found when $\Delta$ is $0.2,35$ rules are built and the constructing network has among the best for which the values of MAE are equal to 0.217 in training, 0.122 in validation, and 0.049 in testing, respectively. Consequently, we suggest that the influence of the $\Delta$ value is set as 0.2 and the network has 6 inputs, 35 hidden nodes, and one output. With small error and not complex structure, the results suggest that the constructed network is suitable and do not have over fitting problem. It is noted that the value of MAE in

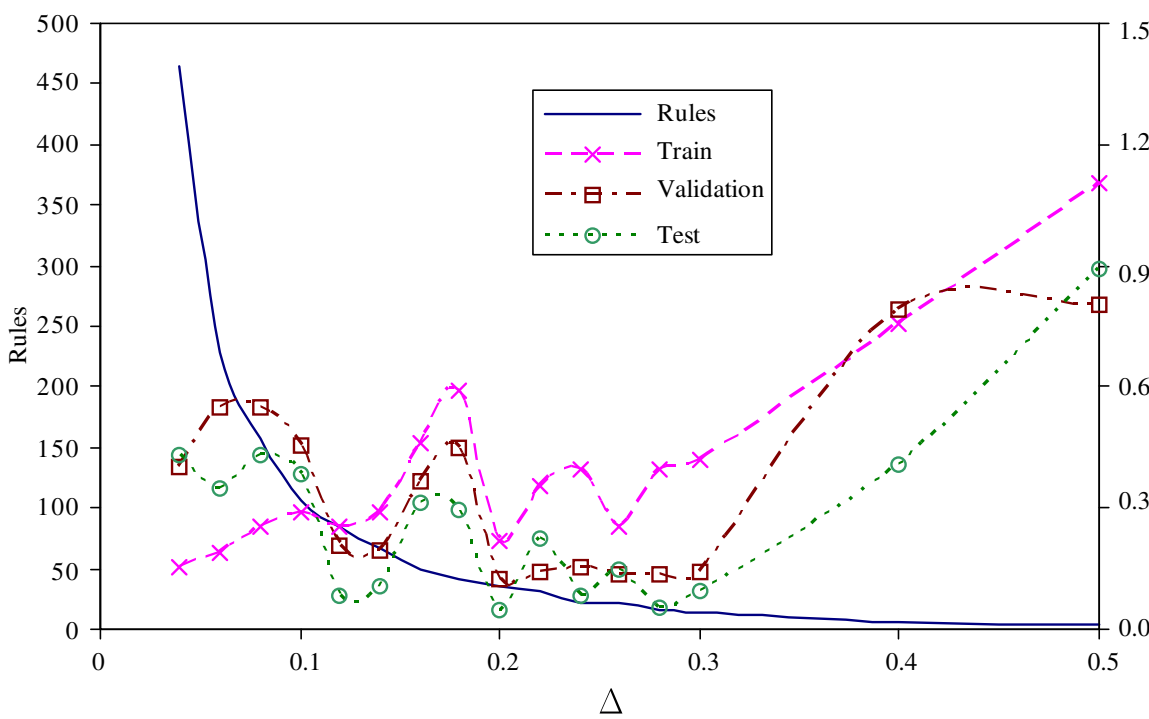

$\stackrel{1}{\Sigma}$

Figure 7 Influence of $\Delta$ value on network modelling.

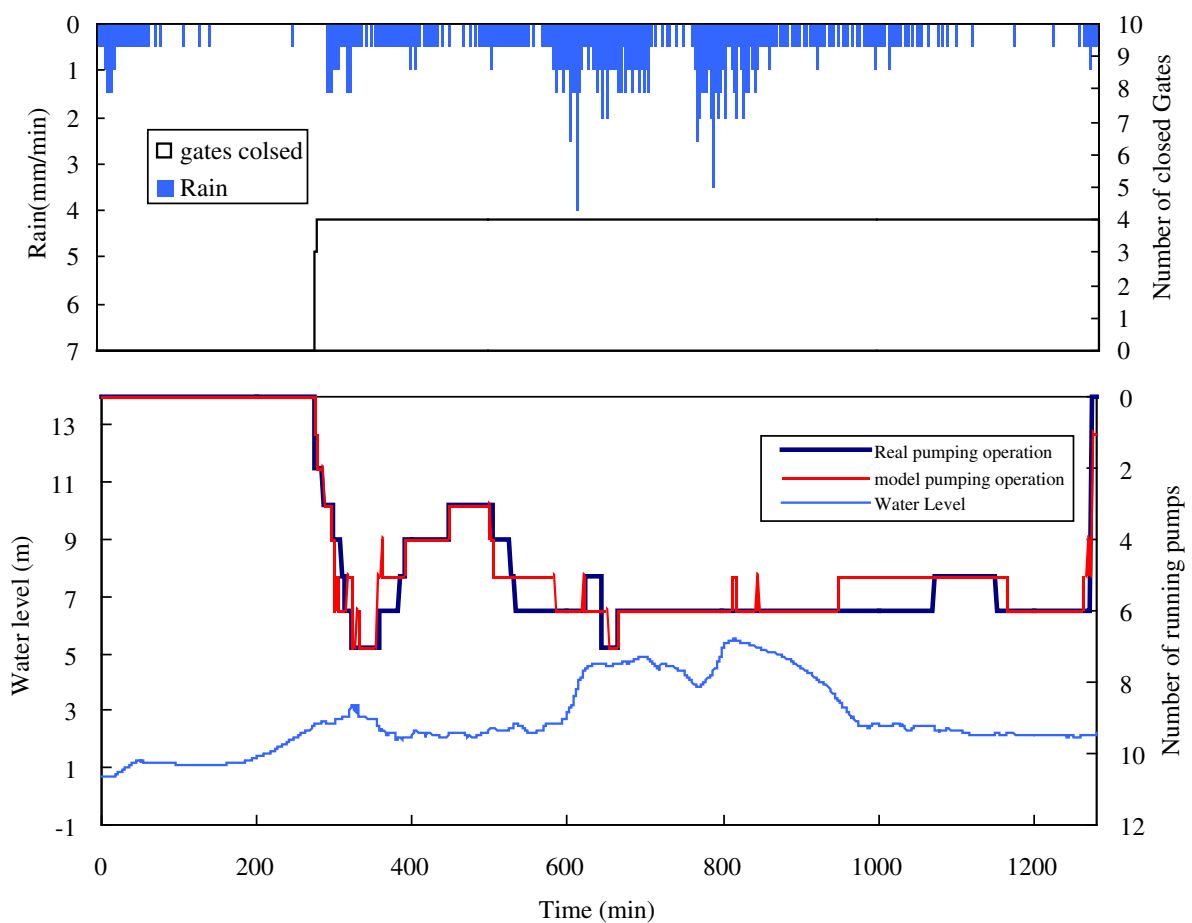

Figure 8 Comparison of model operation and real operation in training stage (Haima Typhoon). 
testing case is much smaller than the value of MAE in training case (also in validation case). This is mainly because the output (the number of active pump) is relatively stable in the validation and testing cases than in the training case (shown in Figs. 8-10).

Fig. 8 shows a comparison between the model output and the actual operating records in a training case (Haima Ty- phoon). It appears the model outputs nicely correspond with the actual operating records. Table 3 gives the analyses of comparing between the numbers of actual opened pumps with the model estimated. In training phase, the accuracy of model estimation for the opened pumping sets less than 4 pumps can reach $91.5 \%$ to $100 \%$, and the errors are all under 1 set. When the number of pump sets is equal or greater

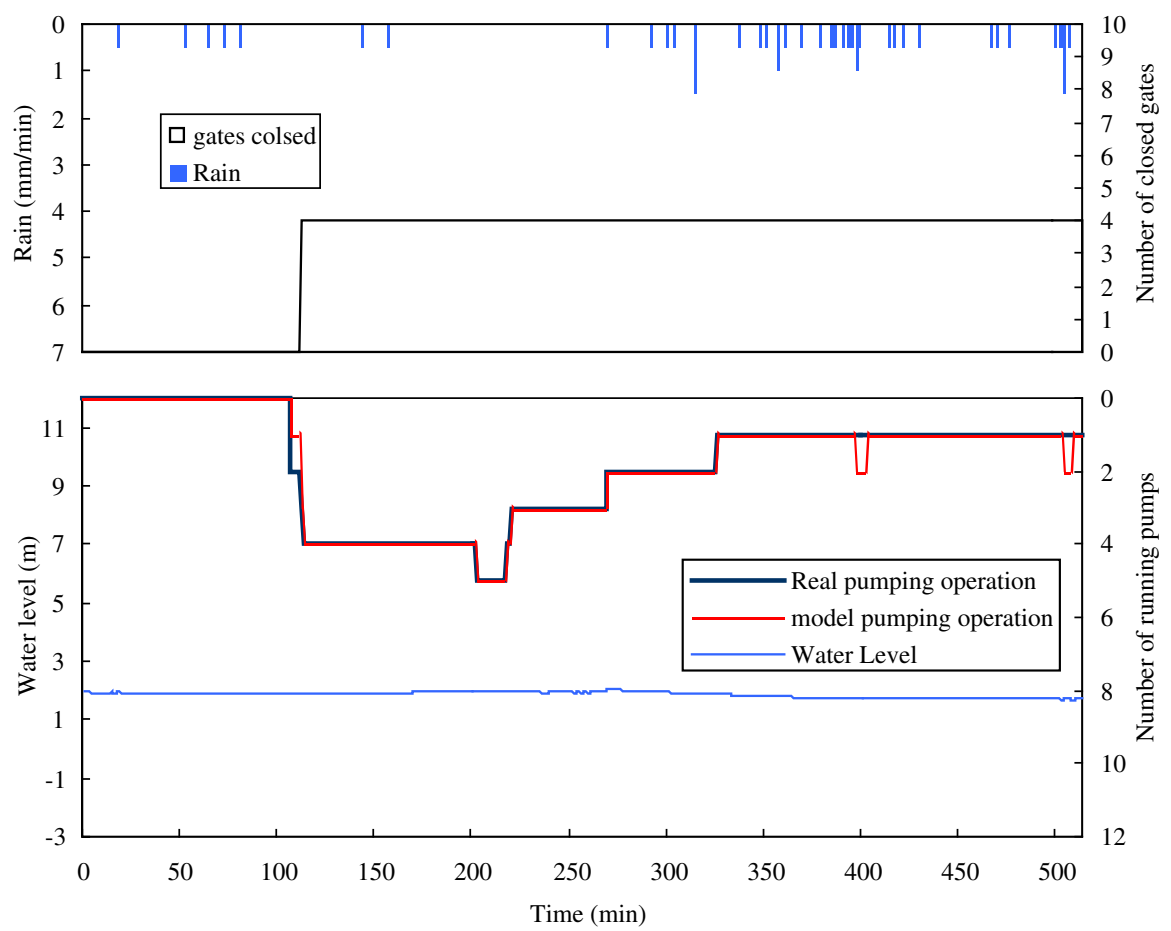

Figure 9 Comparison of model operation and real operation in validation stage (Storm event).
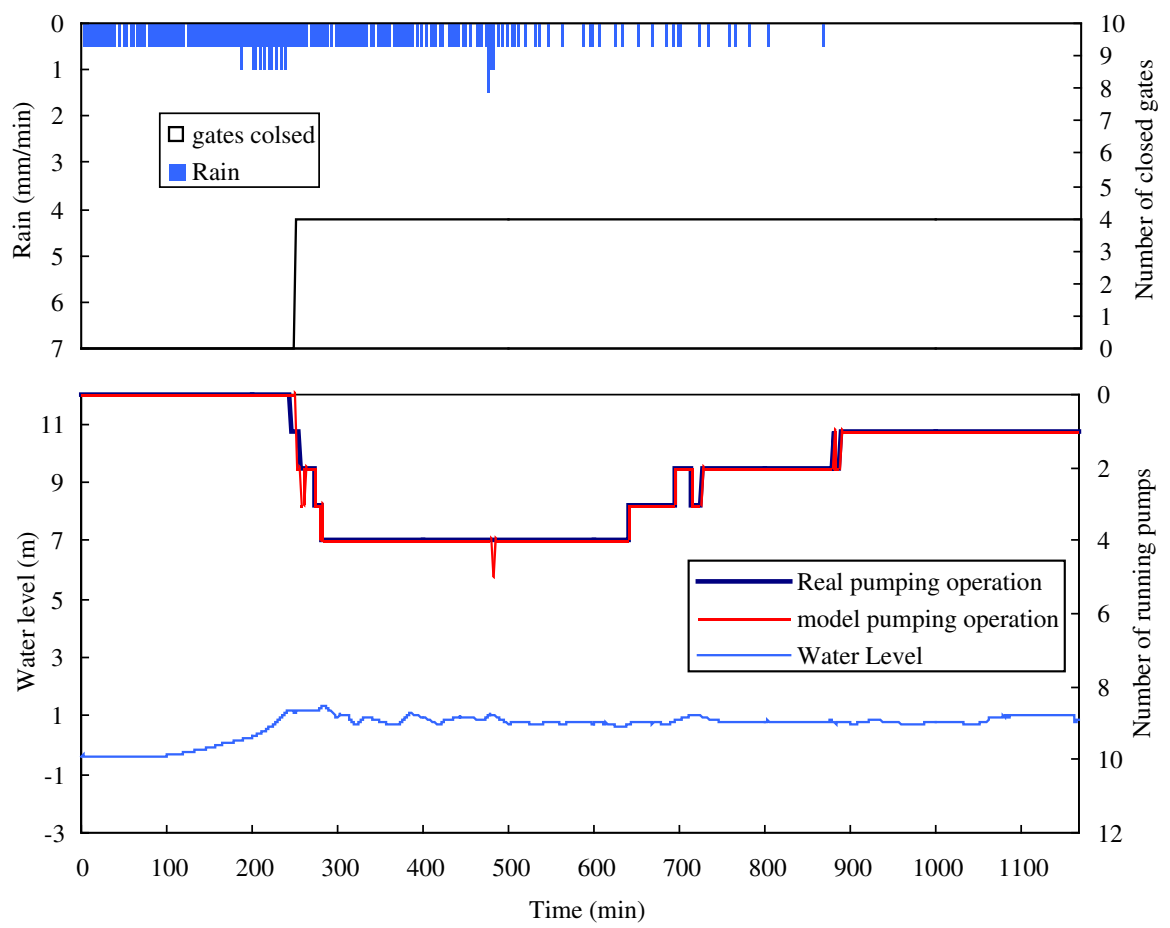

Figure 10 Comparison of model operation and real operation in testing stage (Haitang Typhoon). 
Table 3 Comparison of Model Operation and Real Operation

\begin{tabular}{|c|c|c|c|c|c|c|c|c|c|c|c|c|c|c|c|c|c|}
\hline \multirow[t]{2}{*}{ Pumps } & \multirow[t]{2}{*}{ On (min) } & \multicolumn{15}{|c|}{ Error (Prediction-Observation) } & \multirow{2}{*}{$\frac{\text { Accuracy }}{\%}$} \\
\hline & & -7 & -6 & -5 & -4 & -3 & -2 & -1 & 0 & 1 & 2 & 3 & 4 & 5 & 6 & 7 & \\
\hline \multicolumn{18}{|l|}{ Train } \\
\hline 0 & 1395 & 0 & 0 & 0 & 0 & 0 & 0 & 0 & 1384 & 11 & 0 & 0 & 0 & 0 & 0 & 0 & 99.21 \\
\hline 1 & 467 & 0 & 0 & 0 & 0 & 0 & 0 & 0 & 467 & 0 & 0 & 0 & 0 & 0 & 0 & 0 & 100.00 \\
\hline 2 & 380 & 0 & 0 & 0 & 0 & 0 & 0 & 4 & 348 & 28 & 0 & 0 & 0 & 0 & 0 & 0 & 91.58 \\
\hline 3 & 391 & 0 & 0 & 0 & 0 & 0 & 0 & 0 & 382 & 9 & 0 & 0 & 0 & 0 & 0 & 0 & 97.70 \\
\hline 4 & 102 & 0 & 0 & 0 & 0 & 0 & 0 & 0 & 68 & 27 & 7 & 0 & 0 & 0 & 0 & 0 & 66.67 \\
\hline 5 & 116 & 0 & 0 & 0 & 0 & 0 & 0 & 0 & 93 & 23 & 0 & 0 & 0 & 0 & 0 & 0 & 80.17 \\
\hline 6 & 655 & 0 & 0 & 0 & 0 & 0 & 4 & 233 & 418 & 0 & 0 & 0 & 0 & 0 & 0 & 0 & 63.82 \\
\hline 7 & 59 & 0 & 0 & 0 & 0 & 0 & 6 & 14 & 39 & 0 & 0 & 0 & 0 & 0 & 0 & 0 & 66.10 \\
\hline \multicolumn{18}{|c|}{ Validation } \\
\hline 0 & 107 & 0 & 0 & 0 & 0 & 0 & 0 & 0 & 107 & 0 & 0 & 0 & 0 & 0 & 0 & 0 & 100.00 \\
\hline 1 & 189 & 0 & 0 & 0 & 0 & 0 & 0 & 0 & 178 & 11 & 0 & 0 & 0 & 0 & 0 & 0 & 94.18 \\
\hline 2 & 61 & 0 & 0 & 0 & 0 & 0 & 0 & 0 & 56 & 5 & 0 & 0 & 0 & 0 & 0 & 0 & 91.80 \\
\hline 3 & 51 & 0 & 0 & 0 & 0 & 0 & 0 & 0 & 51 & 0 & 0 & 0 & 0 & 0 & 0 & 0 & 100.00 \\
\hline 4 & 92 & 0 & 0 & 0 & 0 & 0 & 0 & 0 & 92 & 0 & 0 & 0 & 0 & 0 & 0 & 0 & 100.00 \\
\hline 5 & 15 & 0 & 0 & 0 & 0 & 0 & 0 & 0 & 15 & 0 & 0 & 0 & 0 & 0 & 0 & 0 & 100.00 \\
\hline \multicolumn{18}{|l|}{ Test } \\
\hline 0 & 245 & 0 & 0 & 0 & 0 & 0 & 0 & 0 & 245 & 0 & 0 & 0 & 0 & 0 & 0 & 0 & 100.00 \\
\hline 1 & 292 & 0 & 0 & 0 & 0 & 0 & 0 & 5 & 282 & 5 & 0 & 0 & 0 & 0 & 0 & 0 & 96.58 \\
\hline 2 & 198 & 0 & 0 & 0 & 0 & 0 & 0 & 0 & 193 & 5 & 0 & 0 & 0 & 0 & 0 & 0 & 97.47 \\
\hline 3 & 74 & 0 & 0 & 0 & 0 & 0 & 0 & 0 & 73 & 1 & 0 & 0 & 0 & 0 & 0 & 0 & 98.65 \\
\hline 4 & 359 & 0 & 0 & 0 & 0 & 0 & 0 & 0 & 356 & 3 & 0 & 0 & 0 & 0 & 0 & 0 & 99.16 \\
\hline
\end{tabular}

than 4 sets, the accuracy of the estimation becomes lower, from $64 \%$ to $80 \%$.

Figs. 9 and 10 present the number of opened pumps through a typhoon event by the model operation and the real operating record in the validation and testing cases. As shown in Table 3, the accuracies are all higher than 91\% in the validation and testing phases, and the errors are all within 1 pump set. Apparently, the built model could get good results in the validation and testing phases. However, there are no recorded operation events that contain 6 or 7 pumps in validation and testing stages. How well built the model is for the prediction of a high number of active pumps, unfortunately, could not be verified. After carefully examining the results, we found the model's accuracy for 4 and 5 pumps in training phase could only reach $67 \%$ to $80 \%$, while the accuracy in validation and testing phases could reach nearly $100 \%$. Therefore, it is believed that the built network can well estimate with considerable accuracy.

\section{Conclusions}

Taipei City is a basin surrounded by the Danshui River, while the narrow estuary makes it difficult for water in the city to be effectively discharged which leads to flooding. The flood control system is highly dependent on operating pumping stations. Thus, continuous consideration must be given to improving the operation efficiency of existing or newly developed pumping stations. Developing optimal operating rules and linking those with the design system shows much promise. This study presents a human-like operation strategy for the pumping station by using a novel neural-fuzzy network. The proposed CFNN fuses the ANN and fuzzy logic features to tune the complicated conversion of human intelligence to real operating systems. The combination of neural networks and fuzzy logic concepts allows the advantages of each approach to be utilized while, at the same time, overcoming their respective disadvantages. The advantages include simple basic structure, efficient learning ability, ease of expandability, and humanistic clustering effects. The CFNN is a good pattern recognition engine and robust classifier, with the ability to generalize in making decisions about imprecise input data.

The network is implemented in the Yu-Chen pumping station, Taipei. The station contains seven massive pumps. The network was built and verified based on historical typhoon (or storm) events data sets, which include cumulative precipitation, fore bay level, the gravity gates, and operating record of pump sets. A total of 5248 data sets are divided into three independent sets, namely training, verification, and testing. The results demonstrate that the developed intelligent network can provide human-like performance in determining the number of active pumps in the pumping station. The similarity of the active pumps between the network's estimation and historical record, in terms of accuracy, is higher than $91 \%$ in the validation and testing phases, and the estimating errors are all within 1 pump in almost all the cases.

The CFNN has built 35 fuzzy rules to estimate the active pumps based on the related hydrological conditions. We found the accuracy of the forecasting is sensitive to the numbers of rule units. If the number of the rules is increased, the error (in terms of MSE and MAE) will be decreased in the training phase but increased after a certain 
number of rules in the validation and testing phases. The results suggest that 35 rules seem adequate in this study case and more rules could cause over-fitting and diminishing model performance in the testing phase. The study gives an insight into the applicability of fuzzy control to systems with rapidly changing dynamics. It becomes clear that a self-organizing neural-fuzzy network can be efficient to construct a limited number of IF-THEN rules which are likely to respond successfully to the possible input patterns. This gives the CFNN model powerful and accurate forecasting ability.

\section{Acknowledgments}

This study was partially supported by the National Science Council (NSC), Taiwan ROC (NSC 95-2313-b-002-031) and by the Taipei City Government for providing the valuable data sets.

\section{References}

Bae, D.H., Jeong, D.M., Kim, G., 2007. Monthly dam inflow forecasts using weather forecasting information and neuro-fuzzy technique. Hydrological Science Journal 52 (1), 99-113.

Bhattacharya, B., Lobbrecht, A.H., Solomatine, D.P., 2003. Neural networks and reinforcement learning in control of water systems. Journal of Water Resources Planning and Management 129 (6), 458-465.

Chaves, P., Chang, F.J., 2008. Intelligent Reservoir Operation System Based on Evolving Artificial Neural Networks. Advances in Water Resources 31, 926-936.

Chaves, P., Kojiri, T., 2007. Deriving reservoir operational strategies considering water quantity and quality objectives by stochastic fuzzy neural networks. Advances in Water Resources 30 (5), 1329-1341.

Chang, F.J., Chang, Y.T., 2006. Adaptive neuro-fuzzy inference system for prediction of water level in reservoir. Advances in Water Resources 29, 1-10.

Chang, F.-J., Hu, H.-F., Chen, Y.-C., 2001. Counterpropagation fuzzy-neural network for streamflow reconstructing. Hydrological Processes 15 (2), 219-232.

Chang, L.C., Chang, F.J., 2001. Intelligent control for modeling of real-time reservoir operation. Hydrological Processes 15 (9), 1621-1634.
Chang, Y.T., Chang, L.C., Chang, F.J., 2005. Intelligent control for modelling of real-time reservoir operation, part II: artificial neural with operating rule curves. Hydrological Processes 19, 1431-1444.

Hsu, N.S., Wei, C.C., 2007. A multipurpose reservoir real-time operation model for flood control during typhoon invasion. Journal of Hydrology 336 (3-4), 282-293.

Jingyi, Z., Hall, M.J., 2004. Regional flood frequency analysis for the Gan-Ming River basin in China. Journal of Hydrology 296, 98117.

Kohonen, T., 1998. Self-organization and associative memory, second ed. Springer-Verlag, Berlin, Germany.

Lobbrecht, A.H., Solomatine, D.P., 2002. Machine learning in real-time control of water systems. Urban Water 4, 283289.

Lobbrecht, A.H., Yonas, B.D., Solomatine, D.P., 2005. Neural networks and Fuzzy systems in model based control of the Overwaard Polder. Journal of Water Resources Planning and Management 131, 135-145.

Lohani, A.K., Goel, N.K., Bhatia, K.K.S., 2006. Takagi-Sugeno fuzzy inference system for modeling stage-discharge relationship. Journal of Hydrology 331, 146-160.

Manual of Taipei City Raining Drainage Plan, New Construction Office of Public Works Department, Taipei City Government, 1989 (in Chinese).

Nayak, P.C., Sudheer, K.P., Rangan, D.M., Ramasastri, K.S., 2004. A neuro-fuzzy computing technique for modeling hydrological time series. Journal of Hydrology 291 (1-2), 52-66.

Nie, J., 1997. Nonlinear time-series forecasting: a fuzzy-neural approachNeurocomputing, vol. 16. Elsevier, pp. 63-761.

Nie, J., Linkens, D.A., 1994. Fast self-learning multivariable fuzzy controllers constructed from a modified CPN network. International Journal of Control 60 (3), 369-393.

Nie, J., Linkens, D.A., 1995. Fuzzy-Neural Control: Principles, Algorithms and Applications. Prentice-Hall, London.

Pumping Station Operating Manual, Maintenance Office of Public Works Department, Taipei City Government, 1984 (in Chinese).

Taipei City Government, Handouts for Operating and Maintaining Training of Pumping Station Operators, Maintenance Office of Public Works Department, Taipei, 1998 (in Chinese).

Tutmez, B., Hatipoglu, Z., Kaymak, U., 2006. Modelling electrical conductivity of groundwater using an adaptive neuro-fuzzy inference system. Computers and Geosciences 32 (4), 421433.

Xiong, L., Shamseldin, A., O'Connor, K., 2001. A non-linear combination of the forecasts of rainfall-runoff models by the first-order Takagi-Sugeno fuzzy system. Journal of Hydrology 245, 196-217. 